Bull. Austral. Math. Soc.

Vol. 49 (1994) [101-110]

\title{
ON THE DIVERGENCE OF HERMITE-FEJÉR TYPE INTERPOLATION WITH EQUIDISTANT NODES
}

\author{
T.M. Mills AND Simon J. SMith
}

If $f(x)$ is defined on $[-1,1]$, let $\bar{H}_{1 n}(f, x)$ denote the Lagrange interpolation polynomial of degree $n$ (or less) for $f$ which agrees with $f$ at the $n+1$ equally spaced points $x_{k, n}=-1+(2 k) / n(0 \leqslant k \leqslant n)$. A famous example due to $\mathrm{S}$. Bernstein shows that even for the simple function $h(x) \equiv|x|$, the sequence $\bar{H}_{1 n}(h, x)$ diverges as $n \rightarrow \infty$ for each $x$ in $0<|x|<1$. A generalisation of Lagrange interpolation is the Hermite-Fejér interpolation polynomial $\bar{H}_{m n}(f, x)$, which is the unique polynomial of degree no greater than $m(n+1)-1$ which satisfies $\bar{H}_{m n}^{(p)}\left(f, x_{k, n}\right)=\delta_{0, p} f\left(x_{k, n}\right)(0 \leqslant p \leqslant m-1,0 \leqslant k \leqslant n)$. In general terms, if $m$ is an even number, the polynomials $\bar{H}_{m n}(f, x)$ seem to possess better convergence properties than the $\bar{H}_{1 n}(f, x)$. Nevertheless, D.L. Berman was able to show that for $g(x) \equiv x$, the sequence $\bar{H}_{2 n}(g, x)$ diverges as $n \rightarrow \infty$ for each $x$ in $0<|x|$. In this paper we extend Berman's result by showing that for any even $m, \bar{H}_{m n}(g, x)$ diverges as $n \rightarrow \infty$ for each $x$ in $0<|x|<1$. Further, we are able to obtain an estimate for the error $\left|\bar{H}_{m n}(g, x)-g(x)\right|$.

\section{INTRODUCTION}

Suppose $-1 \leqslant x_{0, n}<x_{1, n}<\ldots<x_{n, n} \leqslant 1$ is an arbitrary system of interpolation nodes. (We shall often write $x_{k, n}$ as $x_{k}$.) Let $m \geqslant 1$ be an integer, and suppose $f$ is a real-valued function defined on $[-1,1]$. The $(0,1, \ldots, m-1)$ Hermite-Fejér (HF) interpolation polynomial $\bar{H}_{m n}(f, x)$ for $f$ is the unique polynomial of degree at most $m(n+1)-1$ which satisfies the $m(n+1)$ conditions

$$
\begin{cases}\bar{H}_{m n}\left(f, x_{k}\right)=f\left(x_{k}\right) & (k=0,1, \ldots, n), \\ \bar{H}_{m n}^{(p)}\left(f, x_{k}\right)=0 & (p=1,2, \ldots, m-1 ; k=0,1, \ldots, n) .\end{cases}
$$

Note that $\bar{H}_{1 n}(f, x)$ is the well-known Lagrange interpolation polynomial for $f(x)$. In 1914, Faber [4] showed that for any system of nodes, there exists a function $f(x)$, continuous on $[-1,1]$, such that $\bar{H}_{1 n}(f, x)$ does not converge uniformly to $f(x)$ on $[-1,1]$ as $n \rightarrow \infty$. On the other hand, Fejér [5] showed in 1916 that if

Received 22nd February, 1993.

Copyright Clearance Centre, Inc. Serial-fee code: 0004-9729/94 $\$ A 2.00+0.00$. 
$x_{k}=-\cos (((2 k+1) \pi) /(2 n+2))$ (so the $x_{k}$ are the zeros of the Chebyshev polynomial $\left.T_{n+1}(x)=\cos ((n+1) \arccos x)\right)$, and if $f$ is continuous on $[-1,1]$, then

$$
\lim _{n \rightarrow \infty} \bar{H}_{2 n}(f, x)=f(x),
$$

uniformly in $[-1,1]$. Thus $(0,1)$ HF interpolation seems to possess better convergence properties than Lagrange interpolation.

Fejér's result has prompted many authors to study $(0,1, \ldots, m-1)$ HF interpolation, particularly when the nodes of interpolation are the zeros of some orthogonal polynomials (such as the Chebyshev polynomials). Much less popular has been a study of $(0,1, \ldots, m-1)$ HF interpolation based on the equidistant nodes

$$
x_{k, n}=x_{k}=-1+\frac{2 k}{n} \quad(k=0,1, \ldots, n) .
$$

One reason for the lack of attention paid to equidistant nodes is a result of Bernstein [2], who showed in 1918 that if $h(x) \equiv|x|$, and the $x_{k}$ are given by (1.1), then the sequence $\bar{H}_{1 n}(h, x)$ diverges as $n \rightarrow \infty$ for each $x$ in $0<|x|<1$. Thus Lagrange interpolation on equidistant nodes diverges for a simple function such as $h(x)$. A quantitative version of Bernstein's result was developed by Byrne, Mills and Smith [3], who showed that if $0<|x|<1$, then

$$
\limsup _{n \rightarrow \infty} \frac{1}{n} \log \left|\bar{H}_{1 n}(h, x)-h(x)\right|=\frac{1}{2}[(1+x) \log (1+x)+(1-x) \log (1-x)] .
$$

(See also Li and Mohapatra [6].)

For $(0,1)$ HF interpolation on the equidistant nodes (1.1), Berman [1] showed in 1958 that even for $g(x) \equiv x$, the sequence $\bar{H}_{2 n}(g, x)$ diverges as $n \rightarrow \infty$ for each $x$ in $0<|x|<1$. The only results of this type for $(0,1, \ldots, m-1)$ HF interpolation $(m \geqslant 3)$ that we have been able to locate in the literature are due to Mendelevic [7]. In particular, Mendelevic showed that if $m$ is even, the $(0,1, \ldots, m-1)$ HF interpolation process based on the equidistant nodes $y_{k, n}=k / n(k=1,2, \ldots, n)$ diverges for the function

$$
\Psi(x)= \begin{cases}0 & (0 \leqslant x<1 / 2), \\ x-1 / 2 & (1 / 2 \leqslant x \leqslant 1),\end{cases}
$$

on a set $E \subset[0,1]$, where $E$ has measure greater than 0.26 .

In this paper we shall prove the following theorem that both generalises and quantifies Berman's divergence result, and also provides a simpler example of divergence of $(0,1, \ldots, m-1) \mathrm{HF}$ interpolation on equidistant nodes for even $m$ than Mendelevič's example. 
TheOREM 1. Suppose $m \geqslant 2$ is even, and $g(x) \equiv x$. Then for each $x$ in $0<$ $|x|<1$, the $(0,1, \ldots, m-1)$ Hermite-Fejér interpolation polynomials $\bar{H}_{m n}(g, x)$ based on the equidistant nodes

$$
x_{k, n}=x_{k}=-1+\frac{2 k}{n} \quad(k=0,1, \ldots, n)
$$

satisfy

$$
\limsup _{n \rightarrow \infty} \frac{1}{n} \log \left|\bar{H}_{m n}(g, x)-g(x)\right|=\frac{m}{2}[(1+x) \log (1+x)+(1-x) \log (1-x)] .
$$

The proof of Theorem 1 will be presented in Section 3. We note that since \pm 1 are interpolation nodes for all $n$, then $g(-1)=\bar{H}_{m n}(g,-1)$ and $g(1)=\bar{H}_{m n}(g, 1)$ for all $n$. Furthermore, since the equidistant nodes are distributed symmetrically about 0 , then $\bar{H}_{m n}(f, x)$ is an odd function whenever $f(x)$ is odd. Hence $g(0)=\bar{H}_{m n}(g, 0)=0$ for all $n$. Thus Theorem 1 settles the convergence behaviour of $\bar{H}_{m n}(g, x)$ for all $x$ in $[-1,1]$. We also point out that our proof of Theorem 1 does not readily adapt to the case when $m(\geqslant 3)$ is an odd number. However, we conjecture that Theorem 1 remains true for all such values of $m$. (For $m=1$, Theorem 1 is false, since $\bar{H}_{m n}(g, x) \equiv g(x)$, although (by (1.2)) it does hold true if $g$ is replaced by $h(x) \equiv|x|$.)

\section{Preliminary REsults}

In this section we introduce further notation and some preliminary results that will be needed for the proof of Theorem 1 .

Suppose

$$
-1 \leqslant x_{0, n}<x_{1, n}<\ldots<x_{n, n} \leqslant 1
$$

is a system of interpolatory nodes, and let $m \geqslant 1$ be an integer. If $f(x)$ is $m-1$ times differentiable on $[-1,1]$, the $(0,1, \ldots, m-1)$ Hermite interpolation polynomial for $f$ is the unique polynomial $H_{m n}(f, x)$ of degree $m(n+1)-1$ or less which satisfies

$$
H_{m n}^{(p)}\left(f, x_{k}\right)=f^{(p)}\left(x_{k}\right) \quad(p=0,1, \ldots, m-1 ; k=0,1, \ldots, n) .
$$

$H_{m n}(f, x)$ can be written in the form

$$
H_{m n}(f, x)=\sum_{k=0}^{n} \sum_{j=0}^{m-1} f^{(j)}\left(x_{k}\right) A_{j k}(x),
$$

where the polynomials $A_{j k}(x)$ (more precisely, $A_{j k m n}(x)$ ) are the unique polynomials of degree $m(n+1)-1$ or less which satisfy

$$
A_{j k}^{(p)}\left(x_{q}\right)=\delta_{j p} \delta_{k q} \quad(j, p=0,1, \ldots, m-1 ; k, q=0,1, \ldots, n) .
$$


Note that the $(0,1, \ldots, m-1) \mathrm{HF}$ interpolation polynomial for $f$ can be written as

$$
\bar{H}_{m n}(f, x)=\sum_{k=0}^{n} f\left(x_{k}\right) A_{0 k}(x) \text {. }
$$

Now, if $g(x) \equiv x$, then $H_{m n}(g, x) \equiv x$. Hence, by $(2.2)$,

$$
\sum_{k=0}^{n} x_{k} A_{0 k}(x)+\sum_{k=0}^{n} A_{1 k}(x)=x
$$

for all $x$ in $[-1,1]$, and so by $(2.3)$,

$$
x-\bar{H}_{m n}(g, x)=\sum_{k=0}^{n} A_{1 k}(x) .
$$

Thus to prove (1.3) it will suffice to consider $\sum_{k=0}^{n} A_{1 k}(x)$.

The following formula for the $A_{j k}(x)$ was developed by Szabados [8, Lemma 1]. Define

$$
\omega_{n}(x)=\prod_{k=0}^{n}\left(x-x_{k}\right)
$$

and put

$$
\ell_{k}(x)=\ell_{k n}(x)=\frac{\omega_{n}(x)}{\omega_{n}^{\prime}\left(x_{k}\right)\left(x-x_{k}\right)} \quad(k=0,1, \ldots, n)
$$

Then

(2.7) $A_{j k}(x)=\frac{\ell_{k}(x)^{m}}{j !}\left(x-x_{k}\right)^{j} B_{j k}(x) \quad(j=0,1, \ldots, m-1 ; k=0,1, \ldots, n)$, where

$$
B_{j k}(x)=B_{j k m n}(x)=\sum_{i=0}^{m-j-1} b_{i k}\left(x-x_{k}\right)^{i} \quad(j=0,1, \ldots, m-1 ; k=0,1, \ldots, n),
$$

and

$$
b_{i k}=b_{i k m n}=\frac{\left[\ell_{k}(x)^{-m}\right]_{x=x_{k}}^{(i)}}{i !} \quad(i=0,1, \ldots ; k=0,1, \ldots, n) .
$$

We shall need the following lemma which is also due to Szabados [8, Lemmas 2 and 3].

LEMma 1. Define

$$
a_{i k}=a_{i k m n}=m \sum_{\substack{\nu=0 \\ \nu \neq k}}^{n} \frac{1}{\left(x_{\nu}-x_{k}\right)^{i}} \quad(k=0,1, \ldots, n ; i=1,2, \ldots),
$$


and let $B_{j k}(x)$ and $b_{i k}$ be given by (2.8) and (2.9). Then

$$
b_{i k}=\frac{1}{i} \sum_{\nu=1}^{i} a_{\nu k} b_{i-\nu, k} \quad(k=0,1, \ldots, n ; i=1,2, \ldots) .
$$

Also, there exists a positive number $c$ (depending only on $j$ and $m$ ) so that

$$
\begin{gathered}
B_{j k}(x) \geqslant c\left(\frac{x-x_{k}}{x_{k}-x_{k \neq 1}}\right)^{m-j-1} \\
(-\infty<x<\infty, m-j \text { odd, } 0 \leqslant j \leqslant m-1,0 \leqslant k \leqslant n),
\end{gathered}
$$

with one of the signs in $x_{k \pm 1}$.

The formulas and results of this section so far are valid for an arbitrary system (2.1) of nodes. Henceforth we shall assume that the interpolation nodes are the equidistant nodes

$$
x_{k}=-1+\frac{2 k}{n} \quad(k=0,1, \ldots, n) .
$$

We now develop an upper bound for $\left|B_{1 k}(x)\right|$ which, by (2.4) and (2.7), will be useful later when obtaining bounds for $\left|\bar{H}_{m n}(g, x)-x\right|$.

Lemma 2. There exist constants $c_{i m}(i=0,1, \ldots ; m=1,2, \ldots)$ so that for $n \geqslant 2$,

$$
\left|b_{i k}\right| \leqslant c_{i m}(n \log n)^{i} \quad(i=0,1, \ldots ; k=0,1, \ldots, n) .
$$

PROOF: From (2.10) and (2.13) we have

$$
a_{i k}=m\left(\frac{n}{2}\right)^{i} \sum_{\substack{\nu=0 \\ \nu \neq k}}^{n} \frac{1}{(\nu-k)^{i}} \quad(k=0,1, \ldots, n ; i=1,2, \ldots),
$$

and so

$$
\left|a_{i k}\right| \leqslant m\left(\frac{n}{2}\right)^{i} \times 2 \sum_{r=1}^{n} \frac{1}{r^{i}} \leqslant m n^{i} \sum_{r=1}^{n} \frac{1}{r^{i}} .
$$

Thus there exist constants $c_{m}$ (independent of $n$ ) so that

$$
\left|a_{i k}\right| \leqslant \begin{cases}c_{m} n \log n & (i=1 ; k=0,1, \ldots, n), \\ c_{m} n^{i} & (i=2,3, \ldots ; k=0,1, \ldots, n) .\end{cases}
$$

We now prove (2.14) by induction on $i$. Since $\ell_{k}\left(x_{k}\right)=1$ for all $k,(2.9)$ yields $b_{0 k}=1$ for all $k$, and so (2.14) holds true for $i=0$ if we define $c_{0 m}=1$. If (2.14) holds true for $i=0,1, \ldots, r-1$, then by (2.11) and (2.15) we have 


$$
\begin{aligned}
\left|b_{r k}\right| & \leqslant \frac{1}{r} \sum_{\nu=1}^{r}\left|a_{\nu k}\right|\left|b_{r-\nu, k}\right| \\
& \leqslant \frac{c_{m}}{r}\left(n \log n \times c_{r-1, m}(n \log n)^{r-1}+\sum_{\nu=2}^{r} n^{\nu} \times c_{r-\nu, m}(n \log n)^{r-\nu}\right) \\
& =\frac{c_{m}}{r}(n \log n)^{r}\left(c_{r-1, m}+\sum_{\nu=2}^{r} \frac{c_{r-\nu, m}}{(\log n)^{\nu}}\right) \\
& \leqslant \frac{c_{m}}{r}(n \log n)^{r}\left(c_{r-1, m}+\sum_{\nu=2}^{r} \frac{c_{r-\nu, m}}{(\log 2)^{\nu}}\right) .
\end{aligned}
$$

On defining

$$
c_{r m}=\frac{c_{m}}{r}\left(c_{r-1, m}+\sum_{\nu=2}^{r} \frac{c_{r-\nu, m}}{(\log 2)^{\nu}}\right),
$$

the lemma is established.

Corollary. There exist constants $d_{m}(m=1,2, \ldots)$ so that for $n=2,3, \ldots$,

$$
\left|B_{1 k}(x)\right| \leqslant d_{m}(n \log n)^{m-2} \quad(k=0,1, \ldots, n ;-1 \leqslant x \leqslant 1) .
$$

Proof: Since $\left|x-x_{k}\right| \leqslant 2,(2.8)$ gives

$$
\left|B_{1 k}(x)\right| \leqslant \sum_{i=0}^{m-2} 2^{i}\left|b_{i k}\right|
$$

By Lemma 2 we then have

$$
\begin{aligned}
\left|B_{1 k}(x)\right| & \leqslant \sum_{i=0}^{m-2} 2^{i} c_{i m}(n \log n)^{i} \\
& \leqslant d_{m}(n \log n)^{m-2}
\end{aligned}
$$

where $d_{m}=\sum_{i=0}^{m-2} 2^{i} c_{i m}$.

We consider next the polynomials $\ell_{k}(x)$ as defined by (2.5) and (2.6). Upon writing

$$
x=x_{j}+\frac{2 \theta}{n}=-1+\frac{2}{n}(j+\theta)
$$

where $0 \leqslant \theta<1$, and using (2.13), we obtain

$$
\ell_{k}(x)=\frac{(-1)^{k+j}(\theta)_{j+1}(1-\theta)_{n-j}}{(j+\theta-k) k !(n-k) !}
$$

where

$$
(a)_{k}= \begin{cases}1 & (k=0), \\ a(a+1) \ldots(a+k-1) & (k=1,2, \ldots) .\end{cases}
$$


The following lemma will be useful in determining the behaviour of the $\ell_{k}(x)$ for large $n$.

Lemma 3. If $|x|<1$, and $x$ is given by (2.17) with $0 \leqslant \theta<1$, define

$$
q(x)=q_{n}(x)=(1+\theta)_{j}(2-\theta)_{n-j-1} .
$$

Then

(2.20)

$$
\lim _{n \rightarrow \infty}\left(\frac{1}{n} \log q(x)-\log n\right)=-1-\log 2+\frac{1}{2}[(1+x) \log (1+x)+(1-x) \log (1-x)] .
$$

Proof: Firstly note from (2.17) that

$$
\lim _{n \rightarrow \infty} \frac{j}{n}=\frac{1+x}{2}
$$

and so $j, n-j \rightarrow \infty$ as $n \rightarrow \infty$. Now, (2.19) can be written as

$$
q(x)=\frac{\Gamma(j+1+\theta) \Gamma(n+1-j-\theta)}{\Gamma(1+\theta) \Gamma(2-\theta)},
$$

and hence, upon using the asymptotic expansion [9, page 252]

$$
\log \Gamma(z)=\left(z-\frac{1}{2}\right) \log z-z+\frac{1}{2} \log (2 \pi)+O\left(z^{-1}\right)
$$

as $z \rightarrow \infty$, we obtain

$\frac{1}{n} \log q(x)$

$$
\begin{aligned}
= & \left(\frac{j+1 / 2+\theta}{n}\right) \log (j+1+\theta)+\left(\frac{n+1 / 2-j-\theta}{n}\right) \log (n+1-j-\theta)-1+O\left(n^{-1}\right) \\
= & \left(\frac{j+1 / 2+\theta}{n}\right) \log \left(\frac{j+1+\theta}{n}\right)+\left(\frac{n+1 / 2-j-\theta}{n}\right) \log \left(\frac{n+1-j-\theta}{n}\right) \\
& \quad+\log n-1+O\left(\frac{\log n}{n}\right) .
\end{aligned}
$$

The lemma is then established by letting $n \rightarrow \infty$ and using (2.21).

\section{Proof of the Theorem}

We now prove Theorem 1 . Since $\bar{H}_{m n}(g, x)$ is odd, we can assume without loss of generality that $x<0$. Write $x=-1+2(j+\theta) / n$, where $0 \leqslant \theta<1$. By (2.4) and (2.7) we have

$$
\left|x-\bar{H}_{m n}(g, x)\right|=\left|\sum_{k=0}^{n} \ell_{k}(x)^{m} B_{1 k}(x)\left(x-x_{k}\right)\right| .
$$


Now, from (2.12), $B_{1 k}(x)>0$ for all $x$, and so

$$
\operatorname{sgn}\left[\ell_{k}(x)^{m} B_{1 k}(x)\left(x-x_{k}\right)\right]=\left\{\begin{array}{cc}
+1 & (j \geqslant k) \\
-1 & (j<k)
\end{array}\right.
$$

Therefore, on putting $n^{\prime}=[n / 2]$, we obtain

$$
\begin{gathered}
\ell_{n^{\prime}}(x)^{m} B_{1 n^{\prime}}(x)\left(x_{n^{\prime}}-x\right)-\left(\sum_{k=0}^{j} \ell_{k}(x)^{m} B_{1 k}(x)\left(x-x_{k}\right)\right) \\
\leqslant\left|x-\bar{H}_{m n}(g, x)\right| \leqslant \sum_{k=0}^{n} \ell_{k}(x)^{m} B_{1 k}(x)\left|x-x_{k}\right| .
\end{gathered}
$$

We work firstly with the right hand side of (3.1). Since $\left|x-x_{k}\right| \leqslant 2$, and $k !(n-k) ! \geqslant\left(n^{\prime}\right) !\left(n-n^{\prime}\right) !$, we have from $(2.16)$ and $(2.18)$,

$$
\left|x-\bar{H}_{m n}(g, x)\right| \leqslant \frac{2 d_{m}(n \log n)^{m-2}}{\left(\left(n^{\prime}\right) !\left(n-n^{\prime}\right) !\right)^{m}} \sum_{k=0}^{n}\left(\frac{(\theta)_{j+1}(1-\theta)_{n-j}}{j+\theta-k}\right)^{m} .
$$

Now $(\theta(1-\theta)) /(|j+\theta-k|) \leqslant 1$ for all $k$, and so

$$
\left|x-\bar{H}_{m n}(g, x)\right| \leqslant 2 d_{m}(n+1)(n \log n)^{m-2}\left(\frac{(1+\theta)_{j}(2-\theta)_{n-j-1}}{\left(n^{\prime}\right) !\left(n-n^{\prime}\right) !}\right)^{m} .
$$

Thus, with $q(x)$ given by (2.19), we have

$$
\frac{1}{n} \log \left|x-\bar{H}_{m n}(g, x)\right| \leqslant \frac{m}{n} \log \left(\frac{q(x)}{\left(n^{\prime}\right) !\left(n-n^{\prime}\right) !}\right)+O\left(\frac{\log n}{n}\right) .
$$

From (2.22) it follows that

$$
\frac{1}{n} \log \left(\left(n^{\prime}\right) !\left(n-n^{\prime}\right) !\right)=\log n-\log 2-1+O\left(\frac{\log n}{n}\right) .
$$

Hence, by (2.20), we can conclude from (3.2) that

(3.4) $\limsup _{n \rightarrow \infty} \frac{1}{n} \log \left|x-\bar{H}_{m n}(g, x)\right| \leqslant \frac{m}{2}[(1+x) \log (1+x)+(1-x) \log (1-x)]$.

Next consider the summation term on the left hand side of (3.1). Since $\lim _{n \rightarrow \infty} j / n=$ $(1+x) / 2<1 / 2$, there exists a number $\alpha<1 / 2$ so that $j<\alpha n$ for all $n$ large enough. Then, because $k !(n-k) !=\Gamma(k+1) \Gamma(n-k+1)>\Gamma(\alpha n+1) \Gamma((1-\alpha) n+1)$ for $0 \leqslant$ 
$k \leqslant j$, we have (as with the derivation of (3.2)),

$\frac{1}{n} \log \left|\sum_{k=0}^{j} \ell_{k}(x)^{m} B_{1 k}(x)\left(x-x_{k}\right)\right| \leqslant \frac{m}{n} \log \left(\frac{q(x)}{\Gamma(\alpha n+1) \Gamma((1-\alpha) n+1)}\right)+O\left(\frac{\log n}{n}\right)$.

By (2.22) we can write

$$
\frac{1}{n} \log (\Gamma(\alpha n+1) \Gamma((1-\alpha) n+1))=\log n+\log \left(\alpha^{\alpha}(1-\alpha)^{1-\alpha}\right)-1+O\left(\frac{\log n}{n}\right),
$$

and so by $(2.20)$ we have

$$
\begin{aligned}
\underset{n \rightarrow \infty}{\limsup } \frac{1}{n} \log \left(\sum_{k=0}^{j} \ell_{k}(x)^{m} B_{1 k}(x)\left(x-x_{k}\right)\right) \\
\leqslant \frac{m}{2}[(1+x) \log (1+x)+(1-x) \log (1-x)]+k m,
\end{aligned}
$$

where $k=-\log 2-\log \left(\alpha^{\alpha}(1-\alpha)^{1-\alpha}\right)<0$.

It remains to consider the expression $\ell_{n^{\prime}}(x)^{m} B_{1 n^{\prime}}(x)\left(x_{n^{\prime}}-x\right)$ on the left hand side of (3.1). Because $\left|x_{n^{\prime}}-x_{n^{\prime} \pm 1}\right|=2 / n,(2.12)$ gives

$$
B_{1 n^{\prime}}(x) \geqslant c\left(\frac{n}{2}\right)^{m-2}\left(x_{n^{\prime}}-x\right)^{m-2}=c\left(n^{\prime}-j-\theta\right)^{m-2},
$$

where $c$ depends only on $m$. Thus

$$
\ell_{n^{\prime}}(x)^{m} B_{1 n^{\prime}}(x)\left(x_{n^{\prime}}-x\right) \geqslant \frac{2 c}{n\left(n^{\prime}-j-\theta\right)}\left(\frac{q(x)}{\left(n^{\prime}\right) !\left(n-n^{\prime}\right) !}\right)^{m}(\theta(1-\theta))^{m} .
$$

By Berman [1, Lemma 1] for each $x$ there exists an increasing sequence $\left\{k_{n}\right\}_{n=1}^{\infty}$ of positive integers, and a number $a(x)$ with $0<a(x)<1 / 2$, such that if we write

$$
x=-1+\frac{2}{k_{n}}(j+\theta)
$$

where $0 \leqslant \theta<1$, then $a(x) \leqslant \theta \leqslant 1-a(x)$ for all $n$. Hence we can assume $\theta(1-\theta)$ has a positive lower bound, and then, on using $n^{\prime}-j-\theta \leqslant n,(3.6)$ can be written in the form

$$
\ell_{n^{\prime}}(x)^{m} B_{1 n^{\prime}}(x)\left(x_{n^{\prime}}-x\right) \geqslant \frac{c^{\prime}}{n^{2}}\left(\frac{q(x)}{\left(n^{\prime}\right) !\left(n-n^{\prime}\right) !}\right)^{m}
$$

where $c^{\prime}$ depends only on $m$. By (2.20) and (3.3) we can conclude that

$\limsup _{n \rightarrow \infty} \frac{1}{n} \log \left(\ell_{n^{\prime}}(x)^{m} B_{1 n^{\prime}}(x)\left(x_{n^{\prime}}-x\right)\right) \geqslant \frac{m}{2}[(1+x) \log (1+x)+(1-x) \log (1-x)]$. 
To complete the proof of Theorem 1, we observe that the estimates (3.5) and (3.7) for the terms on the left hand side of (3.1) yield

$$
\limsup _{n \rightarrow \infty} \frac{1}{n} \log \left|x-\bar{H}_{m n}(g, x)\right| \geqslant \frac{m}{2}[(1+x) \log (1+x)+(1-x) \log (1-x)] .
$$

The required statement (1.3) then follows from (3.4) and (3.8).

\section{REFERENCES}

[1] D.L. Berman, 'Divergence of the Hermite-Fejér interpolation process', (in Russian), Uspehi Mat. Nauk. 13 (1958), 143-148.

[2] S. Bernstein, 'Quelques remarques sur l'interpolation', Math. Ann. 79 (1918), 1-12.

[3] G.J. Byrne, T.M. Mills and S.J. Smith, 'On Lagrange interpolation with equidistant nodes', Bull. Austral. Math. Soc. 42 (1990), 81-89.

[4] G. Faber, 'Über die interpolatorische Darstellung stetiger Funktionen', Jahresber. Deutsch. Math. Verein. 23 (1914), 190-210.

[5] L. Fejér, 'Über interpolation', Göttinger Nachrichten (1916), 66-91.

[6] X. Li and R.N. Mohapatra, 'On the divergence of Lagrange interpolation with equidistant nodes', Proc. Amer. Math. Soc. (to appear).

[7] L.B. Mendelevič, 'Divergence of the Hermite interpolation polynomials with multiple equidistant nodes', (in Russian), in Proceedings of the Central Regional Association of Mathematics Departments, Functional Analysis and the Theory of Functions 2 (Kalinin. Gos. Ped. Inst., Kalinin, 1971), pp. 136-142.

[8] J. Szabados, 'On the order of magnitude of fundamental polynomials of Hermite interpolation', Acta Math. Hungar. (to appear).

[9] E.T. Whittaker and G.N. Watson, $A$ course of modern analysis, 4th edition (Cambridge Univ. Press, London, 1973).

Department of Mathematics

La Trobe University, Bendigo

PO Box 199

Bendigo, Vic. 3550

Australia 